Elect. Comm. in Probab. 14 (2009), 347-357

ELECTRONIC

COMMUNICATIONS

in PROBABILITY

\title{
RATE OF ESCAPE OF THE MIXER CHAIN
}

\section{ARIEL YADIN}

Faculty of Mathematics and Computer Science, The Weizmann Institute of Science, Rehovot 76100, Israel.

email: ariel.yadin@weizmann.ac.il

Submitted January 19, 2009, accepted in final form June 4, 2009

AMS 2000 Subject classification: 60J10, 60B15

Keywords: Random walks

\section{Abstract}

The mixer chain on a graph $G$ is the following Markov chain: Place tiles on the vertices of $G$, each tile labeled by its corresponding vertex. A "mixer" moves randomly on the graph, at each step either moving to a randomly chosen neighbor, or swapping the tile at its current position with some randomly chosen adjacent tile.

We study the mixer chain on $\mathbb{Z}$, and show that at time $t$ the expected distance to the origin is $t^{3 / 4}$, up to constants. This is a new example of a random walk on a group with rate of escape strictly between $t^{1 / 2}$ and $t$.

\section{Introduction}

Let $G=(V, E)$ be a graph. On each vertex $v \in V$, place a tile marked $v$. Consider the following Markov chain, which we call the mixer chain. A "mixer" performs a random walk on the graph. At each time step, the mixer chooses a random vertex adjacent to its current position. Then, with probability $1 / 2$ it moves to that vertex, and with probability $1 / 2$ it remains at the current location, but swaps the tiles on the current vertex and the adjacent vertex. If $G$ is the Cayley graph of a group, then the mixer chain turns out to be a random walk on a different group.

Aside from being a canonical process, the mixer chain is interesting because of its rate of escape. The rate of escape is the asymptotic growth of $\mathbb{E}\left[d\left(X_{t}, X_{0}\right)\right]$ as a function of $t$, where $d(\cdot, \cdot)$ is the graphical distance. For a random walk $\left\{X_{t}\right\}$ on some graph $G$, we use the terminology degree of escape for the limit

$$
\lim _{t \rightarrow \infty} \frac{\log \mathbb{E}\left[d\left(X_{t}, X_{0}\right)\right]}{\log t} .
$$

When restricting to random walks on groups, it is still open what values in $[0,1]$ can be obtained by degrees of escape. For example, if the group is $\mathbb{Z}^{d}$ then the degree of escape is $1 / 2$. On a $d$-ary tree (free group) the degree of escape is 1 . As far as the author is aware, the only other examples known were given by Erschler in [1] (see also [4]). Erschler iterates a construction known as the lamp-lighter (slightly similar to the mixer chain), and produces examples of groups with degrees of escape $1-2^{-k}, k=1,2, \ldots$, . 
After formally defining the mixer chain on general groups, we study the mixer chain on $\mathbb{Z}$. Our main result, Theorem 2.1 , shows that the mixer chain on $\mathbb{Z}$ has degree of escape $3 / 4$.

It is not difficult to show (perhaps using ideas from this note) that on transient groups the mixer chain has degree of escape 1 . Since all recurrent groups are essentially $\mathbb{Z}$ and $\mathbb{Z}^{2}$, it seems that the mixer chain on other groups cannot give examples of other degrees of escape. As for $\mathbb{Z}^{2}$, one can show that the mixer chain has degree of escape 1 . In fact, the ideas in this note suggest that the distance to the origin in the mixer chain on $\mathbb{Z}^{2}$ is $n \log ^{-1 / 2}(n)$ up to constants, (we conjecture that this is the case). For the reader interested in logarithmic corrections to the rate of escape, in [2] Erschler gave examples of rates of escape that are almost linear with a variety of logarithmic corrections. Logarithmic corrections are interesting because linear rate of escape is equivalent to the the existence of non-constant bounded harmonic functions, to non-trivial Poisson boundary, and to the positivity of the associated entropy, see [3].

After introducing some notation, we provide a formal definition of the mixer chain as random walk on a Cayley graph. The generalization to general graphs is immediate.

Acknowledgements. I wish to thank Itai Benjamini for suggesting this construction, and for useful discussions. I also wish to thank an anonymous referee for pointing out useful references.

\subsection{Notation}

Let $G$ be a group and $U$ a generating set for $G$, such that if $x \in U$ then $x^{-1} \in U$ ( $U$ is called symmetric). The Cayley graph of $G$ with respect to $U$ is the graph with vertex set $G$ and edge set $\left\{\{g, h\}: g^{-1} h \in U\right\}$. Let $\mathscr{D}$ be a distribution on $U$. Then we can define the random walk on $G$ (with respect to $U$ and $\mathscr{D}$ ) as the Markov chain with state space $G$ and transition matrix $P(g, h)=1\left\{g^{-1} h \in U\right\} \mathscr{D}\left(g^{-1} h\right)$. We follow the convention that such a process starts from the identity element in $G$.

A permutation of $G$ is a bijection from $G$ to $G$. The support of a permutation $\sigma$, denoted $\operatorname{supp}(\sigma)$, is the set of all elements $g \in G$ such that $\sigma(g) \neq g$. Let $\Sigma$ be the group of all permutations of $G$ with finite support (multiplication is composition of functions). By $<g, h>$ we denote the transposition of $g$ and $h$; that is, the permutation $\sigma$ with support $\{g, h\}$ such that $\sigma(g)=h$, $\sigma(h)=g$. By $<g_{1}, g_{2}, \ldots, g_{n}>$ we denote the cyclic permutation $\sigma$ with support $\left\{g_{1}, \ldots, g_{n}\right\}$, such that $\sigma\left(g_{j}\right)=g_{j+1}$ for $j<n$ and $\sigma\left(g_{n}\right)=g_{1}$.

For an element $g \in G$ we associate a canonical permutation, denoted by $\phi_{g}$, defined by $\phi_{g}(h)=g h$ for all $h \in G$. It is straightforward to verify that the map $g \mapsto \phi_{g}$ is a homomorphism of groups, and so we use $g$ to denote $\phi_{g}$. Although $g \notin \Sigma$, we have that $g \sigma g^{-1} \in \Sigma$ for all $\sigma \in \Sigma$.

We now define a new group, that is in fact the semi-direct product of $G$ and $\Sigma$, with respect to the homomorphism $g \mapsto \phi_{g}$ mentioned above. The group is denoted by $G \ltimes \Sigma$, and its elements are $G \times \Sigma$. Group multiplication is defined by:

$$
(g, \sigma)(h, \tau) \stackrel{\text { def }}{=}\left(g h, g \tau g^{-1} \sigma\right) .
$$

We leave it to the reader to verify that this is a well-defined group operation. Note that the identity element in this group is $(e, \mathbf{i d})$, where id is the identity permutation in $\Sigma$ and $e$ is the identity element in $G$. Also, the inverse of $(g, \sigma)$ is $\left(g^{-1}, g^{-1} \sigma^{-1} g\right)$.

We use $d(g, h)=d_{G, U}(g, h)$ to denote the distance between $g$ and $h$ in the group $G$ with respect to the generating set $U$; i.e., the minimal $k$ such that $g^{-1} h=\prod_{j=1}^{k} u_{j}$ for some $u_{1}, \ldots, u_{k} \in U$. The generating set also provides us with a graph structure. $g$ and $h$ are said to be adjacent if $d(g, h)=1$, that is if $g^{-1} h \in U$. A path $\gamma$ in $G$ (with respect to the generating set $U$ ) is a sequence 
$\left(\gamma_{0}, \gamma_{1}, \ldots, \gamma_{n}\right) \cdot|\gamma|$ denotes the length of the path, which is defined as the length of the sequence minus 1 (in this case $|\gamma|=n$ ).

\subsection{Mixer Chain}

In order to define the mixer chain we require the following

Proposition 1.1. Let $U$ be a finite symmetric generating set for $G$. Then,

$$
\Upsilon=\{(u, \mathbf{i d}),(e,<e, u>): u \in U\}
$$

generates $G \ltimes \Sigma$. Furthermore, for any cyclic permutation $\sigma=<g_{1}, \ldots, g_{n}>\in \Sigma$,

$$
d_{G \ltimes \Sigma, \Upsilon}\left(\left(g_{1}, \sigma\right),\left(g_{1}, \mathbf{i d}\right)\right) \leq 5 \sum_{j=1}^{n} d\left(g_{j}, \sigma\left(g_{j}\right)\right) .
$$

Proof. Let $D((g, \sigma),(h, \tau))$ denote the minimal $k$ such that $(g, \sigma)^{-1}(h, \tau)=\prod_{j=1}^{k} v_{j}$, for some $v_{1}, \ldots, v_{k} \in \Upsilon$, with the convention that $D((g, \sigma),(h, \tau))=\infty$ if there is no such finite sequence of elements of $\Upsilon$. Thus, we want to prove that $D((g, \sigma),(e, \mathbf{i d}))<\infty$ for all $g \in G$ and $\sigma \in \Sigma$. Note that by definition for any $f \in G$ and $\pi \in \Sigma, D((g, \sigma),(h, \tau))=D((f, \pi)(g, \sigma),(f, \pi)(h, \tau))$.

A generator simple path in $G$ is a finite sequence of generators $u_{1}, \ldots, u_{k} \in U$ such that for any $1 \leq \ell \leq k, \prod_{j=\ell}^{k} u_{j} \neq e$. By induction on $k$, one can show that for any $k \geq 1$, and for any generator simple path $u_{1}, \ldots, u_{k}$,

$$
\left(e,<e, \prod_{j=1}^{k} u_{j}>\right)=\left(\prod_{j=1}^{k-1}\left(e,<e, u_{j}>\right)\left(u_{j}, \mathbf{i d}\right)\right) \cdot\left(e,<e, u_{k}>\right) \cdot\left(\prod_{j=1}^{k-1}\left(e,<e, u_{k-j}^{-1}>\right)\left(u_{k-j}^{-1}, \mathbf{i d}\right)\right) .
$$

If $d(g, h)=k$ then there exists a generator simple path $u_{1}, \ldots, u_{k}$ such that $h=g \prod_{j=1}^{k} u_{j}$. Thus, we get that for any $h \in G$,

$$
D((e,<e, h>),(e, \mathbf{i d})) \leq 4 d(h, e)-3 .
$$

Because $g<e, g^{-1} h>g^{-1}=<g, h>$, we get that if $\tau=<g, h>\sigma$ then

$$
D((g, \tau),(g, \sigma))=D\left((g, \sigma)\left(e,<e, g^{-1} h>\right),(g, \sigma)(e, \mathbf{i d})\right) \leq 4 d\left(g^{-1} h, e\right)-3=4 d(g, h)-3 .
$$

The triangle inequality now implies that $D((h, \tau),(g, \sigma)) \leq 5 d(g, h)-3$.

Thus, if $\sigma=<g_{1}, g_{2}, \ldots, g_{n}>$, since $\sigma=<g_{1}, g_{2}><g_{2}, g_{3}>\cdots<g_{n-1}, g_{n}>$, we get that

$$
D\left(\left(g_{1}, \sigma\right),\left(g_{1}, \mathbf{i d}\right)\right) \leq 5 \sum_{j=1}^{n-1} d\left(g_{j}, g_{j+1}\right)+d\left(g_{n}, g_{1}\right) .
$$

The proposition now follows from the fact that any $\sigma \in \Sigma$ can be written as a finite product of cyclic permutations.

We are now ready to define the mixer chain: 
Definition 1.2. Let $G$ be a group with finite symmetric generating set $U$. The mixer chain on $G$ (with respect to $U$ ) is the random walk on the group $G \ltimes \Sigma$ with respect to uniform measure on the generating set $\Upsilon=\{(u, \mathbf{i d}),(e,<e, u>): u \in U\}$.

An equivalent way of viewing this chain is viewing the state $(g, \sigma) \in G \ltimes \Sigma$ as follows: The first coordinate corresponds to the position of the mixer on $G$. The second coordinate corresponds to the placing of the different tiles, so the tile marked $x$ is placed on the vertex $\sigma(x)$. By Definition 1.2 , the mixer chooses uniformly an adjacent vertex of $G$, say $h$. Then, with probability $1 / 2$ the mixer swaps the tiles on $h$ and $g$, and with probability $1 / 2$ it moves to $h$. The identity element in $G \ltimes \Sigma$ is (e,id), so the mixer starts at $e$ with all tiles on their corresponding vertices (the identity permutation).

\subsection{Distance Bounds}

In this section we show that the distance of an element in $G \ltimes \Sigma$ to $(e$, id) is essentially governed by the sum of the distances of each individual tile to its origin.

Let $(g, \sigma) \in G \ltimes \Sigma$. Let $\gamma=\left(\gamma_{0}, \gamma_{1}, \ldots, \gamma_{n}\right)$ be a finite path in $G$. We say that the path $\gamma$ covers $\sigma$ if $\operatorname{supp}(\sigma) \subset\left\{\gamma_{0}, \gamma_{1}, \ldots, \gamma_{n}\right\}$. The covering number of $g$ and $\sigma$, denoted $\operatorname{Cov}(g, \sigma)$, is the minimal length of a path $\gamma$, starting at $g$, that covers $\sigma$; i.e.

$$
\operatorname{Cov}(g, \sigma)=\min \left\{|\gamma|: \gamma_{0}=g \text { and } \gamma \text { is a path covering } \sigma\right\} .
$$

To simplify notation, we denote $D=d_{G \ltimes \Sigma, \Upsilon}$.

Proposition 1.3. Let $(g, \sigma) \in G \ltimes \Sigma$. Then,

$$
D((g, \sigma),(g, \mathbf{i d})) \leq 2 \operatorname{Cov}(g, \sigma)+5 \sum_{h \in \operatorname{supp}(\sigma)} d(h, \sigma(h)) .
$$

Proof. The proof of the proposition is by induction on the size of $\operatorname{supp}(\sigma)$. If $|\operatorname{supp}(\sigma)|=0$, then $\sigma=$ id so the proposition holds. Assume that $|\operatorname{supp}(\sigma)|>0$.

Let $n=\operatorname{Cov}(g, \sigma)$, and let $\gamma$ be a path in $G$ such that $|\gamma|=n, \gamma_{0}=g$ and $\gamma$ covers $\sigma$. Write $\sigma=c_{1} c_{2} \cdots c_{k}$, where the $c_{j}$ 's are cyclic permutations with pairwise disjoint non-empty supports, and

$$
\operatorname{supp}(\sigma)=\bigcup_{j=1}^{k} \operatorname{supp}\left(c_{j}\right) .
$$

Let

$$
j=\min \left\{m \geq 0: \gamma_{m} \in \operatorname{supp}(\sigma)\right\} .
$$

So, there is a unique $1 \leq \ell \leq k$ such that $\gamma_{j} \in \operatorname{supp}\left(c_{\ell}\right)$. Let $\tau=c_{\ell}^{-1} \sigma$. Thus,

$$
\operatorname{supp}(\tau)=\bigcup_{j \neq \ell} \operatorname{supp}\left(c_{j}\right)
$$

and specifically, $|\operatorname{supp}(\tau)|<|\operatorname{supp}(\sigma)|$. Note that $h \in \operatorname{supp}\left(\gamma_{j}^{-1} c_{\ell} \gamma_{j}\right)$ if and only if $\gamma_{j} h \in \operatorname{supp}\left(c_{\ell}\right)$, and specifically, $e \in \operatorname{supp}\left(\gamma_{j}^{-1} c_{\ell} \gamma_{j}\right) . \gamma_{j}^{-1} c_{\ell} \gamma_{j}$ is a cyclic permutation, so by Proposition 1.1, we know that

$$
\begin{aligned}
D\left(\left(\gamma_{j}, \sigma\right),\left(\gamma_{j}, \tau\right)\right) & =D\left(\left(\gamma_{j}, \tau\right)\left(e, \gamma_{j}^{-1} c_{\ell} \gamma_{j}\right),\left(\gamma_{j}, \tau\right)\right)=D\left(\left(e, \gamma_{j}^{-1} c_{\ell} \gamma_{j}\right),(e, \mathbf{i d})\right) \\
& \leq 5 \sum_{h \in \operatorname{supp}\left(c_{\ell}\right)} d\left(\gamma_{j}^{-1} h, \gamma_{j}^{-1} c_{\ell}(h)\right)=5 \sum_{h \in \operatorname{supp}\left(c_{\ell}\right)} d(h, \sigma(h)) .
\end{aligned}
$$


By induction,

$$
D\left(\left(\gamma_{j}, \tau\right),\left(\gamma_{j}, \mathbf{i d}\right)\right) \leq 2 \operatorname{Cov}\left(\gamma_{j}, \tau\right)+5 \sum_{h \in \operatorname{supp}(\tau)} d(h, \tau(h))
$$

Let $\beta$ be the path $\left(\gamma_{j}, \gamma_{j+1}, \ldots, \gamma_{n}\right)$. Since $\gamma_{j}$ is the first element in $\gamma$ that is in $\operatorname{supp}(\sigma)$, we get that $\operatorname{supp}(\tau) \subset \operatorname{supp}(\sigma) \subseteq\left\{\gamma_{j}, \gamma_{j+1}, \ldots, \gamma_{n}\right\}$, which implies that $\beta$ is a path of length $n-j$ that covers $\tau$, so $\operatorname{Cov}\left(\gamma_{j}, \tau\right) \leq n-j$. Combining (1.3) and (1.4) we get,

$$
\begin{aligned}
D((g, \sigma),(g, \mathbf{i d})) & \leq D\left(\left(\gamma_{0}, \sigma\right),\left(\gamma_{j}, \sigma\right)\right)+D\left(\left(\gamma_{j}, \sigma\right),\left(\gamma_{j}, \tau\right)\right)+D\left(\left(\gamma_{j}, \tau\right),\left(\gamma_{j}, \text { id }\right)\right)+D\left(\left(\gamma_{j}, \mathbf{i d}\right),\left(\gamma_{0}, \mathbf{i d}\right)\right) \\
& \leq j+5 \sum_{h \in \operatorname{supp}\left(c_{\ell}\right)} d(h, \sigma(h))+5 \sum_{h \in \operatorname{supp}(\tau)} d(h, \sigma(h))+2(n-j)+j \\
& =2 \operatorname{Cov}(g, \sigma)+5 \sum_{h \in \operatorname{supp}(\sigma)} d(h, \sigma(h)) .
\end{aligned}
$$

Proposition 1.4. Let $(g, \sigma) \in G \ltimes \Sigma$ and let $g^{\prime} \in G$. Then,

$$
D\left((g, \sigma),\left(g^{\prime}, \mathbf{i d}\right)\right) \geq \frac{1}{2} \sum_{h \in \operatorname{supp}(\sigma)} d(h, \sigma(h)) .
$$

Proof. The proof is by induction on $D=D\left((g, \sigma),\left(g^{\prime}\right.\right.$,id $\left.)\right)$. If $D=0$ then $\sigma=\mathbf{i d}$, and we are done. Assume that $D>0$. Let $v \in \Upsilon$ be a generator such that $D\left((g, \sigma) v,\left(g^{\prime}, \mathbf{i d}\right)\right)=D-1$. There exists $u \in U$ such that either $v=(u$, id $)$ or $v=(e,<e, u>)$. If $v=(u$, id $)$ then by induction

$$
D \geq D\left((g, \sigma) v,\left(g^{\prime}, \text { id }\right)\right) \geq \frac{1}{2} \sum_{h \in \operatorname{supp}(\sigma)} d(h, \sigma(h)) .
$$

So assume that $v=(e,<e, u>)$. If $\sigma(h) \notin\{g, g u\}$, then $<g, g u>\sigma(h)=\sigma(h)$, and

$$
\operatorname{supp}(\sigma) \backslash\left\{\sigma^{-1}(g), \sigma^{-1}(g u)\right\}=\operatorname{supp}(<g, g u>\sigma) \backslash\left\{\sigma^{-1}(g), \sigma^{-1}(g u)\right\} .
$$

Since $d(g, g u)=1$,

$$
\begin{aligned}
\sum_{h \in \operatorname{supp}(\sigma)} d(h, \sigma(h))= & d\left(g, \sigma^{-1}(g)\right)+d\left(g u, \sigma^{-1}(g u)\right)+\sum_{h \notin\left\{\sigma^{-1}(g), \sigma^{-1}(g u)\right\}} d(h, \sigma(h)) \\
\leq & d(g, g u)+d\left(g u, \sigma^{-1}(g)\right)+d(g u, g)+d\left(g, \sigma^{-1}(g u)\right) \\
& +\sum_{h \notin\left\{\sigma^{-1}(g), \sigma^{-1}(g u)\right\}} d(h,<g, g u>\sigma(h)) \\
\leq & 2+\sum_{h \in \operatorname{supp}(<g, g u>\sigma)} d(h,<g, g u>\sigma(h)) .
\end{aligned}
$$

So by induction,

$$
\begin{aligned}
D & =1+D\left((g,<g, g u>\sigma),\left(g^{\prime}, \mathbf{i d}\right)\right) \geq 1+\frac{1}{2} \sum_{h \in \operatorname{supp}(<g, g u>\sigma)} d(h,<g, g u>\sigma(h)) \\
& \geq \frac{1}{2} \sum_{h \in \operatorname{supp}(\sigma)} d(h, \sigma(h)) .
\end{aligned}
$$




\section{The Mixer Chain on $\mathbb{Z}$}

We now consider the mixer chain on $\mathbb{Z}$, with $\{1,-1\}$ as the symmetric generating set. We denote by $\left\{\omega_{t}=\left(S_{t}, \sigma_{t}\right)\right\}_{t \geq 0}$ the mixer chain on $\mathbb{Z}$.

For $\omega \in \mathbb{Z} \ltimes \Sigma$ we denote by $D(\omega)$ the distance of $\omega$ from $(0$,id) (with respect to the generating set $\Upsilon$, see Definition 1.2). Denote by $D_{t}=D\left(\omega_{t}\right)$ the distance of the chain at time $t$ from the origin.

As stated above, we show that the mixer chain on $\mathbb{Z}$ has degree of escape $3 / 4$. In fact, we prove slightly stronger bounds on the distance to the origin at time $t$.

Theorem 2.1. Let $D_{t}$ be the distance to the origin of the mixer chain on $\mathbb{Z}$. Then, there exist constants $c, C>0$ such that for all $t \geq 0, c t^{3 / 4} \leq \mathbb{E}\left[D_{t}\right] \leq C t^{3 / 4}$.

The proof of Theorem 2.1 is in Section 3 .

For $z \in \mathbb{Z}$, denote by $X_{t}(z)=\left|\sigma_{t}(z)-z\right|$, the distance of the tile marked $z$ to its origin at time $t$. Define

$$
X_{t}=\sum_{z \in \mathbb{Z}} X_{t}(z),
$$

which is a finite sum for any given $t$. As shown in Propositions 1.3 and $1.4, X_{t}$ approximates $D_{t}$ up to certain factors.

For $z \in \mathbb{Z}$ define

$$
V_{t}(z)=\sum_{j=0}^{t} 1\left\{S_{t}=\sigma_{t}(z)\right\} .
$$

$V_{t}(z)$ is the number of times that the mixer visits the tile marked $z$, up to time $t$.

\subsection{Distribution of $X_{t}(z)$}

The following proposition states that the "mirror image" of the mixer chain has the same distribution as the original chain. We omit a formal proof, as the proposition follows from the symmetry of the walk.

Proposition 2.2. For $\sigma \in \Sigma$ define $\sigma^{\prime} \in \Sigma$ by $\sigma^{\prime}(z)=-\sigma(-z)$ for all $z \in \mathbb{Z}$. Then, for any $t \geq 1$, $\left(\left(S_{1}, \sigma_{1}\right), \ldots,\left(S_{t}, \sigma_{t}\right)\right)$ and $\left(\left(-S_{1}, \sigma_{1}^{\prime}\right), \ldots,\left(-S_{t}, \sigma_{t}^{\prime}\right)\right)$ have the same distribution.

By a lazy random walk on $\mathbb{Z}$, we refer to the integer valued process $W_{t}$, such that $W_{t+1}-W_{t}$ are i.i.d. random variables with the distribution $\mathbb{P}\left[W_{t+1}-W_{t}=1\right]=\mathbb{P}\left[W_{t+1}-W_{t}=1\right]=1 / 4$ and $\mathbb{P}\left[W_{t+1}-W_{t}=0\right]=1 / 2$.

Lemma 2.3. Let $t \geq 0$ and $z \in \mathbb{Z}$. Let $k \geq 1$ be such that $\mathbb{P}\left[V_{t}(z)=k\right]>0$. Then, conditioned on $V_{t}(z)=k$, the distribution of $\sigma_{t}(z)-z$ is the same as $W_{k-1}+B$, where $\left\{W_{k}\right\}$ is a lazy random walk on $\mathbb{Z}$ and $B$ is a random variable independent of $\left\{W_{k}\right\}$ such that $|B| \leq 2$.

Essentially the proof is as follows. We consider the successive time at which the mixer visits the tile marked $z$. The movement of the tile at these times is a lazy random walk with the number of steps equal to the number of visits. The difference between the position of the tile at time $t$ and its position at the last visit is at most 1 , and the difference between the tile at time 0 and its position at the first visit is at most $1 . B$ is the random variable that measures these two differences.

Proof. Define inductively the following random times: $T_{0}(z)=0$, and for $j \geq 1$,

$$
T_{j}(z)=\inf \left\{t \geq T_{j-1}(z)+1: S_{t}=\sigma_{t}(z)\right\}
$$


Claim 2.4. Let $T=T_{1}(0)$. For all $\ell$ such that $\mathbb{P}[T=\ell]>0$,

$$
\mathbb{P}\left[\sigma_{T}(0)=1 \mid T=\ell\right]=\mathbb{P}\left[\sigma_{T}(0)=-1 \mid T=\ell\right]=1 / 4,
$$

and

$$
\mathbb{P}\left[\sigma_{T}(0)=0 \mid T=\ell\right]=1 / 2,
$$

Proof. Note that $\left|S_{1}-\sigma_{1}(0)\right|=1$ and that for all $1 \leq t<T, \sigma_{t}(0)=\sigma_{1}(0)$. Thus, $\sigma_{T-1}(0)=\sigma_{1}(0)$ and $S_{T-1}=S_{1}$. So we have the equality of events

$$
\{T=\ell\}=\bigcap_{t=1}^{\ell-1}\left\{S_{t} \neq \sigma_{t}(0)\right\} \bigcap\left\{S_{\ell-1}=S_{1}, \sigma_{\ell-1}(0)=\sigma_{1}(0)\right\} \bigcap\left\{S_{\ell}=\sigma_{1}(0) \text { or } \sigma_{\ell}(0)=S_{1}\right\} \text {. }
$$

Hence, if we denote $\mathscr{E}=\bigcap_{t=1}^{\ell-1}\left\{S_{t} \neq \sigma_{t}(0)\right\} \bigcap\left\{S_{\ell-1}=S_{1}, \sigma_{\ell-1}(0)=\sigma_{1}(0)\right\}$, then

$$
\begin{aligned}
\mathbb{P}[T=\ell] & =\mathbb{P}[\mathscr{E}] \cdot \mathbb{P}\left[S_{\ell}=\sigma_{1}(0) \text { or } \sigma_{\ell}(0)=S_{1} \mid \mathscr{E}\right] \\
& =\mathbb{P}[\mathscr{E}] \cdot \frac{1}{2} .
\end{aligned}
$$

Since the events $\left\{S_{1}=0\right\}$ and $\left\{\sigma_{1}(0)=0\right\}$ are disjoint and their union is the whole space, we get that

$$
\begin{aligned}
\mathbb{P}\left[\sigma_{T}(0)=0, T=\ell\right]= & \mathbb{P}\left[\mathscr{E}, S_{\ell}=\sigma_{1}(0)=0\right]+\mathbb{P}\left[\mathscr{E}, \sigma_{\ell}(0)=S_{1}=0\right] \\
= & \mathbb{P}\left[\mathscr{E}, \sigma_{1}(0)=0\right] \cdot \mathbb{P}\left[S_{\ell}=\sigma_{1}(0) \mid S_{\ell-1}=S_{1}, \sigma_{\ell-1}(0)=\sigma_{1}(0)=0\right] \\
& +\mathbb{P}\left[\mathscr{E}, S_{1}(0)=0\right] \cdot \mathbb{P}\left[\sigma_{\ell}(0)=S_{1} \mid S_{\ell-1}=S_{1}=0, \sigma_{\ell-1}(0)=\sigma_{1}(0)\right] \\
= & \mathbb{P}[\mathscr{E}] \cdot \frac{1}{4} .
\end{aligned}
$$

Combining (2.1) and (2.2) we get that

$$
\mathbb{P}\left[\sigma_{T}(0)=0 \mid T=\ell\right]=\frac{1}{2}
$$

Finally, by Proposition 2.2 ,

$$
\begin{aligned}
\mathbb{P}\left[\sigma_{T}(0)=1, T=\ell\right] & =\mathbb{P}\left[\mathscr{E}, S_{\ell}=\sigma_{\ell}(0)=1\right] \\
& =\mathbb{P}\left[\sigma_{T}(0)=-1, T=\ell\right] .
\end{aligned}
$$

Since the possible values for $\sigma_{T}(0)$ are $-1,0,1$, the claim follows.

We continue with the proof of Lemma 2.3 .

We have the equality of events $\left\{V_{t}(z)=k\right\}=\left\{T_{k}(z) \leq t<T_{k+1}(z)\right\}$.

Let $t_{1}, t_{2}, \ldots, t_{k}, t_{k+1}$ be such that

$$
\mathbb{P}\left[T_{1}(z)=t_{1}, \ldots, T_{k+1}(z)=t_{k+1}\right]>0,
$$

and condition on the event $\mathscr{E}=\left\{T_{1}(z)=t_{1}, \ldots, T_{k+1}(z)=t_{k+1}\right\}$. Assume further that $t_{k} \leq t<$ $t_{k+1}$, so that $V_{t}(z)=k$. Write

$$
\sigma_{t}(z)-z=\sigma_{t}(z)-\sigma_{T_{k}(z)}(z)+\sum_{j=2}^{k} \sigma_{T_{j}(z)}(z)-\sigma_{T_{j-1}(z)}(z)+\sigma_{T_{1}(z)}(z)-z .
$$


For $1 \leq j \leq k-1$ denote $Y_{j}=\sigma_{T_{j+1}(z)}(z)-\sigma_{T_{j}(z)}(z)$. By Claim 2.4 and the Markov property, conditioned on $\mathscr{E},\left\{Y_{j}\right\}$ are independent with the distribution $\mathbb{P}\left[Y_{j}=1 \mid \mathscr{E}\right]=\mathbb{P}\left[Y_{j}=-1 \mid \mathscr{E}\right]=1 / 4$ and $\mathbb{P}\left[Y_{j}=0 \mid \mathscr{E}\right]=1 / 2$. So conditioned on $\mathscr{E}, \sum_{j=1}^{k-1} Y_{j}$ has the same distribution of $W_{k-1}$.

Finally, $\left|\sigma_{t}(z)-\sigma_{T_{k}(z)}(z)\right| \leq 1$, and $\left|\sigma_{T_{1}(z)}(z)-z\right| \leq 1$. Since conditioned on $\mathscr{E}, \sigma_{t}(z)-\sigma_{T_{k}(z)}(z)$, and $\sigma_{T_{1}(z)}(z)-z$ are independent of $\left\{Y_{j}\right\}$, this completes the proof of the lemma.

Corollary 2.5. There exist constants $c, C>0$ such that for all $t \geq 0$ and all $z \in \mathbb{Z}$,

$$
c \mathbb{E}\left[\sqrt{V_{t}(z)}\right]-2 \mathbb{P}\left[V_{t}(z) \geq 1\right] \leq \mathbb{E}\left[X_{t}(z)\right] \leq C \mathbb{E}\left[\sqrt{V_{t}(z)}\right]+2 \mathbb{P}\left[V_{t}(z) \geq 1\right] .
$$

Proof. Let $\left\{W_{t}\right\}$ be a lazy random walk on $\mathbb{Z}$. Note that $\left\{2 W_{t}\right\}$ has the same distribution as $\left\{S_{2 t}^{\prime}\right\}$ where $\left\{S_{t}^{\prime}\right\}$ is a simple random walk on $\mathbb{Z}$. It is well known (see e.g. [5]), that there exist universal constants $c_{1}, C_{1}>0$ such that for all $t \geq 0$,

$$
c_{1} \sqrt{t} \leq \mathbb{E}\left[\left|S_{2 t}^{\prime}\right|\right]=2 \mathbb{E}\left[\left|W_{t}\right|\right] \leq C_{1} \sqrt{t} .
$$

By Lemma 2.3, we know that for any $k \geq 0$,

$$
\mathbb{E}\left[\left|W_{k}\right|\right]-2 \leq \mathbb{E}\left[X_{t}(z) \mid V_{t}(z)=k+1\right] \leq \mathbb{E}\left[\left|W_{k}\right|\right]+2 .
$$

Thus, summing over all $k$, there exists constants $c_{2}, C_{2}>0$ such that

$$
c_{2} \mathbb{E}\left[\sqrt{V_{t}(z)}\right]-2 \mathbb{P}\left[V_{t}(z) \geq 1\right] \leq \mathbb{E}\left[X_{t}(z)\right] \leq C_{2} \mathbb{E}\left[\sqrt{V_{t}(z)}\right]+2 \mathbb{P}\left[V_{t}(z) \geq 1\right]
$$

Lemma 2.6. Let $\left\{S_{t}^{\prime}\right\}$ be a simple random walk on $\mathbb{Z}$ started at $S_{0}^{\prime}=0$, and let

$$
L_{t}(z)=\sum_{j=0}^{t} 1\left\{S_{j}^{\prime}=z\right\}
$$

Then, for any $z \in \mathbb{Z}$, and any $k \in \mathbb{N}$,

$$
\mathbb{P}\left[L_{2 t}(2 z) \geq k\right] \leq \mathbb{P}\left[V_{t}(z) \geq k\right] .
$$

Specifically, $\mathbb{E}\left[\sqrt{L_{2 t}(2 z)}\right] \leq \mathbb{E}\left[\sqrt{V_{t}(z)}\right]$.

Proof. Fix $z \in \mathbb{Z}$. For $t \geq 0$ define $M_{t}=S_{t}-\sigma_{t}(z)+z$. Note that

$$
V_{t}(z)=\sum_{j=0}^{t} \mathbf{1}\left\{M_{j}=z\right\}
$$

so $V_{t}(z)$ is the number of times $\left\{M_{t}\right\}$ visits z up to time $t$.

$\left\{M_{t}\right\}$ is a Markov chain on $\mathbb{Z}$ with the following step distribution.

$$
\mathbb{P}\left[M_{t+1}=M_{t}+\varepsilon \mid M_{t}\right]=\left\{\begin{array}{rrl}
1 / 2 & M_{t}=z, & \varepsilon \in\{-1,1\}, \\
1 / 2 & \left|M_{t}-z\right|=1, & \varepsilon=-M_{t}+z, \\
1 / 4 & \left|M_{t}-z\right|=1, & \varepsilon=0 \\
1 / 4 & \left|M_{t}-z\right|=1, & \varepsilon=M_{t}-z, \\
1 / 4 & \left|M_{t}-z\right|>1, & \varepsilon \in\{-1,1\}, \\
1 / 2 & \left|M_{t}-z\right|>1, & \varepsilon=0 .
\end{array}\right.
$$


Specifically, $\left\{M_{t}\right\}$ is simple symmetric when at $z$, lazy symmetric when not adjacent to $z$, and has a drift towards $z$ when adjacent to $z$.

Define $\left\{N_{t}\right\}$ to be the following Markov chain on $\mathbb{Z}: N_{0}=0$, and for all $t \geq 0$,

$$
\mathbb{P}\left[N_{t+1}=N_{t}+\varepsilon \mid N_{t}\right]=\left\{\begin{array}{lll}
1 / 2 & N_{t}=z, & \varepsilon \in\{-1,1\}, \\
1 / 2 & N_{t} \neq z, & \varepsilon=0, \\
1 / 4 & N_{t} \neq z, & \varepsilon \in\{-1,1\} .
\end{array}\right.
$$

So $\left\{N_{t}\right\}$ is simple symmetric at $z$, and lazy symmetric when not at $z$. Let

$$
V_{t}^{\prime}(z)=\sum_{j=0}^{t} 1\left\{N_{j}=z\right\},
$$

be the number of times $\left\{N_{t}\right\}$ visits $z$ up to time $t$.

Define inductively $\rho_{0}=\rho_{0}^{\prime}=0$ and for $j \geq 0$,

$$
\begin{aligned}
& \rho_{j+1}=\min \left\{t \geq 1: M_{\rho_{j}+t}=z\right\}, \\
& \rho_{j+1}^{\prime}=\min \left\{t \geq 1: N_{\rho_{j}^{\prime}+t}=z\right\} .
\end{aligned}
$$

If $N_{t} \geq M_{t}>z$ then

$$
\mathbb{P}\left[M_{t+1}=M_{t}+1 \mid M_{t}\right]=\mathbb{P}\left[N_{t+1}=N_{t}+1 \mid N_{t}\right],
$$

and

$$
\mathbb{P}\left[M_{t+1}=M_{t}-1 \mid M_{t}\right] \geq \mathbb{P}\left[N_{t+1}=N_{t}-1 \mid N_{t}\right] .
$$

Thus, we can couple $M_{t+1}$ and $N_{t+1}$ so that $M_{t+1} \leq N_{t+1}$. Similarly, if $N_{t} \leq M_{t}<z$ then $M_{t+1}$ moves towards $z$ with higher probability than $N_{t+1}$, and they both move away from $z$ with probability $1 / 4$. So we can couple $M_{t+1}$ and $N_{t+1}$ so that $M_{t+1} \geq N_{t+1}$. If $N_{t}=M_{t}=z$ then $M_{t+1}$ and $N_{t+1}$ have the same distribution, so they can be coupled so that $N_{t+1}=M_{t+1}$. Thus, we can couple $\left\{M_{t}\right\}$ and $\left\{N_{t}\right\}$ so that for all $j \geq 0, \rho_{j} \leq \rho_{j}^{\prime}$ a.s.

Let $\left\{S_{t}^{\prime}\right\}$ be a simple random walk on $\mathbb{Z}$. For $x \in \mathbb{Z}$, let

$$
\tau_{x}=\min \left\{2 t \geq 2: S_{2 t}^{\prime}=2 z, S_{0}^{\prime}=2 x\right\} .
$$

That is, $\tau_{x}$ is the first time a simple random walk started at $2 x$ hits $2 z$ (this is necessarily an even number). In [5, Chapter 9] it is shown that $\tau_{x}$ has the same distribution as $\tau_{2 z}-2|z-x|$. Note that if $N_{t} \neq z$ then $S_{2 t+2}^{\prime}-S_{2 t}^{\prime}$ has the same distribution as $2\left(N_{t+1}-N_{t}\right)$. Since $\left|N_{\rho_{j-1}^{\prime}+1}-z\right|=1$, we get that for all $j \geq 2, \rho_{j}^{\prime}$ has the same distribution as $\frac{1}{2}\left(\tau_{2 z}-2\right)+1$. Also, $\rho_{1}^{\prime}$ has the same distribution as $\frac{1}{2} \tau_{0}$ if $z \neq 0$, and the same distribution as $\frac{1}{2}\left(\tau_{2 z}-2\right)+1$ if $z=0$. Hence, we conclude that for any $k \geq 1, \sum_{j=1}^{k} \rho_{j}^{\prime}$ has the same distribution as $\frac{1}{2} \sum_{j=1}^{k} \tilde{\rho}_{j}$, where $\left\{\tilde{\rho}_{j}\right\}_{j \geq 1}$ are defined by

$$
\tilde{\rho}_{j+1}=\min \left\{2 t \geq 2: S_{\tilde{\rho}_{j}+2 t}^{\prime}=2 z\right\} \text {. }
$$

Finally note that $V_{t}(z) \geq k$ if and only if $\sum_{j=1}^{k} \rho_{j} \leq t, V_{t}^{\prime}(z) \geq k$ if and only if $\sum_{j=1}^{k} \rho_{j}^{\prime} \leq t$, and $L_{t}(2 z) \geq k$ if and only if $\sum_{j=1}^{k} \tilde{\rho}_{j} \leq t$. Thus, under the above coupling, for all $t \geq 0, V_{t}(z) \geq V_{t}^{\prime}(z)$ a.s. Also, $V_{t}^{\prime}(z)$ has the same distribution as $L_{2 t}(2 z)$. The lemma follows. 


\subsection{The Expectation of $X_{t}$}

Recall that $X_{t}=\sum_{z} X_{t}(z)$.

Lemma 2.7. There exists constants $c, C>0$ such that for all $t \geq 0$,

$$
c t^{3 / 4} \leq \mathbb{E}\left[X_{t}\right] \leq C t^{3 / 4} \text {. }
$$

Proof. We first prove the upper bound. For $z \in \mathbb{Z}$ let $A(z)$ be the indicator of the event that the mixer reaches $z$ up to time $t$; i.e. $A_{t}(z)=1\left\{V_{t}(z) \geq 1\right\}$. Note that $\left(\sigma_{t}(z)-z\right)\left(1-A_{t}(z)\right)=0$. Also, by definition $\sum_{z} V_{t}(z)=t$. By Corollary 2.5, using the Cauchy-Schwartz inequality,

$$
\begin{aligned}
\mathbb{E}\left[X_{t}\right] & =\sum_{z} \mathbb{E}\left[X_{t}(z)\right] \leq C_{1} \sum_{z} \mathbb{E}\left[\sqrt{V_{t}(z)}\right]+2 \mathbb{E} \sum_{z} A_{t}(z) \\
& \leq C_{1} \mathbb{E} \sqrt{\sum_{z} V_{t}(z) \cdot \sum_{z} A_{t}(z)}+2 \mathbb{E} \sum_{z} A_{t}(z),
\end{aligned}
$$

for some constant $C_{1}>0$. For any $z \in \mathbb{Z}$, if $A_{t}(z)=1$, then there exists $0 \leq j \leq t$ such that $\left|S_{j}-z\right|=1$. That is, $A_{t}(z)=1$ implies that $z \in\left[m_{t}-1, M_{t}+1\right]$, where $M_{t}=\max _{0 \leq j \leq t} S_{j}$ and $m_{t}=\min _{0 \leq j \leq t} S_{j}$. Thus, $\sum_{z} A(z) \leq M_{t}-m_{t}+2$. Since $M_{t}-m_{t}$ is just the number of sites visited by a lazy random walk, we get (see e.g. [5]) $\mathbb{E}\left[\sum_{z} A_{t}(z)\right] \leq C_{2} \sqrt{t}$, for some constant $C_{2}>0$. Hence, there exists some constant $C_{3}>0$ such that

$$
\mathbb{E}\left[X_{t}\right] \leq C_{1} \sqrt{t \cdot C_{2} \sqrt{t}}+2 C_{2} \sqrt{t} \leq C_{3} t^{3 / 4}
$$

This proves the upper bound.

We turn to the lower bound. Let $\left\{S_{t}^{\prime}\right\}$ be a simple random walk on $\mathbb{Z}$ started at $S_{0}^{\prime}=0$, and let

$$
L_{t}(z)=\sum_{j=0}^{t} \mathbf{1}\left\{S_{j}^{\prime}=z\right\} .
$$

Let

$$
T(z)=\min \left\{t \geq 0: S_{t}^{\prime}=z\right\}
$$

By the Markov property,

$$
\mathbb{P}\left[L_{2 t}(z) \geq k\right] \geq \mathbb{P}[T(z) \leq t] \mathbb{P}\left[L_{t}(0) \geq k\right]
$$

so

$$
\mathbb{E}\left[\sqrt{L_{2 t}(2 z)}\right] \geq \mathbb{P}[T(2 z) \leq t] \mathbb{E}\left[\sqrt{L_{t}(0)}\right]
$$

Theorem 9.3 of [5] can be used to show that $\mathbb{E}\left[\sqrt{L_{t}(0)}\right] \geq c_{1} t^{1 / 4}$, for some constant $c_{1}>0$. By Corollary 2.5, and Lemma 2.6, there exists a constant $c_{2}>0$ such that

$$
\begin{aligned}
\mathbb{E}\left[X_{t}\right] & \geq c_{2} \sum_{z} \mathbb{E}\left[\sqrt{L_{2 t}(2 z)}\right]-2 \sum_{z} A_{t}(z) \\
& \geq c_{1} t^{1 / 4} \cdot c_{2} \mathbb{E} \sum_{z} 1\{T(2 z) \leq t\}-2 C_{2} \sqrt{t}
\end{aligned}
$$

Let $M_{t}^{\prime}=\max _{0 \leq j \leq t} S_{j}^{\prime}$ and $m_{t}^{\prime}=\min _{0 \leq j \leq t} S_{j}^{\prime}$. Then,

$$
\sum_{z} 1\{T(2 z) \leq t\}=\left[m_{t}^{\prime}, M_{t}^{\prime}\right] \bigcap 2 \mathbb{Z}
$$


So for some constants $c_{3}, c_{4}>0$,

$$
\mathbb{E}\left[X_{t}\right] \geq c_{3} t^{1 / 4} \cdot \frac{1}{2} \mathbb{E}\left[M_{t}^{\prime}-m_{t}^{\prime}-1\right]-2 C_{2} \sqrt{t} \geq c_{4} t^{3 / 4}
$$

\section{Proof of Theorem 2.1}

Proof. Recall that $\operatorname{Cov}(z, \sigma)$ is the minimal length of a path on $\mathbb{Z}$, started at $z$, that covers $\operatorname{supp}(\sigma)$. Let $M_{t}=\max _{0 \leq j \leq t} S_{j}$ and $m_{t}=\min _{0 \leq j \leq t} S_{j}$, and let $I_{t}=\left[m_{t}-1, M_{t}+1\right]$. Note that $\operatorname{supp}\left(\sigma_{t}\right) \subset I_{t}$. So for any $z \in I_{t}, \operatorname{Cov}\left(z, \sigma_{t}\right) \leq 2\left|I_{t}\right| .\left\{S_{t}\right\}$ has the distribution of a lazy random walk on $\mathbb{Z}$, so $\left\{2 S_{t}\right\}$ has the same distribution as $\left\{S_{2 t}^{\prime}\right\}$, where $\left\{S_{t}^{\prime}\right\}$ is a simple random walk on $\mathbb{Z}$. It is well known (see e.g. [5, Chapter 2]) that there exist constants $c_{1}, C_{1}>0$ such that $c_{1} \sqrt{t} \leq \mathbb{E}\left[\left|I_{t}\right|\right] \leq C_{1} \sqrt{t}$. Since $S_{t} \in I_{t}$, we get that $\mathbb{E}\left[\operatorname{Cov}\left(S_{t}, \sigma_{t}\right)\right] \leq 2 C_{1} \sqrt{t}$. Together with Propositions 1.3 and 1.4 , and with Lemma 2.7, we get that there exist constants $c, C>0$ such that for all $t \geq 0$,

$$
c t^{3 / 4} \leq \frac{1}{2} \mathbb{E}\left[X_{t}\right] \leq \mathbb{E}\left[D_{t}\right] \leq 2 \mathbb{E}\left[\operatorname{Cov}\left(S_{t}, \sigma_{t}\right)\right]+5 \mathbb{E}\left[X_{t}\right] \leq C t^{3 / 4}
$$

\section{References}

[1] Erschler (Dyubina), A. On the Asymptotics of the Drift. Journal of Mathematical Sciences 121 (2004), 2437-2440. MR1879073

[2] Erschler, A. On Drift And Entropy Growth For Random Walks On Groups. Annals of Probability 31 (2003), 1193-Ü1204.

[3] Kaimanovich, V. A. And Vershik, A. M. Random Walks on Discrete Groups: Boundary and Entropy. Annals of Probability 11 (1983), 457-490. MR0704539

[4] Revelle, D. Rate of Escape of Random Walks on Wreath Products and Related Groups. Annals of Probability 31 (2003), 1917 Ü-1934.

[5] RÉvÉsz, P. Random Walk in Random and Non-Random Environments. World Scientific Publishing Co., 2005. MR2168855 